\title{
Persönlichkeitsschutz in der Online-Welt
}

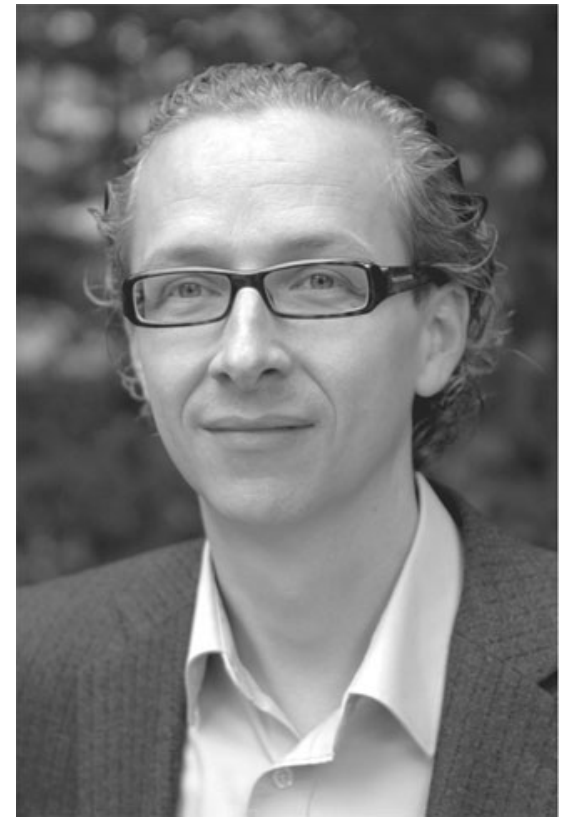

Im Datenschutzrecht ist immer wieder viel darüber diskutiert worden, ob die größere Bedrohung für unsere informationelle Selbstbestimmung vom großen Bruder Staat oder seinen "kleinen Schwestern", den Privatunternehmen, ausgeht. Spätestens seit den Snowden-Enthüllungen wissen wir, dass eine solche Diskussion nur noch wenig zielführend ist. Wir müssen davon ausgehen, dass all das, was Google, Facebook \& Co über uns wissen, stets auch potentiell staatliches Wissen bedeutet. Auch wenn sich daher die Beiträge in diesem Heft in erster Linie mit dem Persönlichkeitsschutz gegenüber nichtstaatlichen Stellen beschäftigen, schwingt doch bei vielen der dort diskutierten Fragestellungen diese überwachungsstaatliche Komponente mit. Der große Datenpool der Online-Welt lässt Differenzierungen nach privaten und staatlichen Datenbanken nicht länger zu, wirksamer Schutz vor staatlicher Überwachung setzt gerade auch einen effektiven Persönlichkeitsschutz gegenüber privaten Unternehmen voraus.

Neben dem Persönlichkeitsschutz im datenschutzrechtlichen Sinne geht es in diesem Heft darüber hinaus auch um den Schutz vor klassischen Persönlichkeitsrechtsverletzungen wie Beleidigungen oder Mobbing im Netz. Das Recht muss hier überzeugende Antworten finden, wie das Persönlichkeitsrecht des Einzelnen auch unter den besonderen Rahmenbedingungen der Online-Welt effektiv geschützt werden kann.

Wolfgang Däubler zeichnet in seinem Beitrag typische Konfliktfelder des Persönlichkeitsschutzes von Arbeitnehmern im Internet nach. Johannes Caspar zeigt auf, wie mühsam der Kampf für einen effektiven Datenschutz gerade im Falle von Sozialen Netzwerken ist. Die internationale Dimension der datenschutzrechtlichen Herausforderungen führt uns Marie-Theres Tinnefeld in ihrem Beitrag vor Augen. Sven Venzke-Caprarese stellt das Social Media Monitoring vor und erläutert dessen rechtliche Rahmenbedingungen. Einen umfassenden Überblick über die typischen Fragestellungen bei Online-Persönlichkeitsrechtsverletzungen gibt Matthias Dittmayer. Dennis Kipker und Friederike Voskamp schließlich wenden sich in ihrem Beitrag dem Thema des Internets als virtuellem Pranger zu.

Ergänzt wird das Schwerpunktthema Persönlichkeitsschutz in diesem Heft durch zwei Beiträge, in denen es um die Themen E-Health und Auftragsdatenverarbeitung geht. Jürgen Kühling und Manuel Klar mahnen für den Datenschutz bei E-Health grundlegende Reformen an und Matthias Bergt zeigt auf, wie Auftragsdatenverarbeitung auch im Massengeschäft rechtskonform gestaltet werden kann.

\section{Benedikt Buchner}

